\title{
An identity involving the mean value of two-term character sums
}

Xiaoyan Guo* and Jingzhe Wang

*Correspondence: guoxiaoyan510@sohu.com Department of Mathematics, Northwest University, Xi'an, Shaanxi, P.R. China

\begin{abstract}
The main purpose of this paper is, using the properties of Gauss sums and the estimate for character sums, to study the mean value problem of the two-term character sums and give an interesting identity for it.
\end{abstract}

MSC: $11 \mathrm{M} 20$

Keywords: two-term character sums; mean value; identity; analytic method; Gauss sums

\section{Introduction}

Let $q \geq 3$ be an integer, $\chi$ be a non-principal character mod $q$. For any integer $n$ with $(n, q)=1$, the two-term character sums $N(n, r, s, \chi ; q)$ are defined as

$$
N(n, r, s, \chi ; q)=\sum_{a=1}^{q} \chi\left(a^{r}+n a^{s}\right)
$$

where $r>s$ are two fixed positive integers. These sums play a very important role in the study of analytic number theory, so they caused many number theorists' interest and favor. Some works related to $N(n, r, s, \chi ; q)$ can be found in [1-4]. If fact, the sums $N(n, r, s, \chi ; q)$ is a special case of the general character sums of the polynomials

$$
\sum_{a=N+1}^{N+M} \chi(f(a))
$$

where $M$ and $N$ are any positive integers, and $f(x)$ is a polynomial. If $q=p$ is an odd prime, then Weil (see [1]) obtained the following important conclusion: Let $\chi$ be a $q$ th-order character $\bmod p$. If $f(x)$ is not a perfect $q$ th power $\bmod p$, then we have the estimate

$$
\sum_{x=N+1}^{N+M} \chi(f(x)) \ll p^{\frac{1}{2}} \ln p,
$$

where '«' constant depends only on the degree of $f(x)$.

Now we are concerned about whether there exists a computational formula for the mean value

$$
\sum_{\chi \bmod }^{*}\left|\sum_{a=1}^{q} \chi\left(a^{r}+n a^{s}\right)\right|^{2 k}
$$


where $s>r \geq 1$ are two integers. In this paper, we shall use the analytic method and the properties of Gauss sums to study this problem, and give an exact computational formula for (3). That is, we shall prove the following theorem.

Theorem 1 Let $q>1$ be an odd integer. Then, for any real number $k$ and integer $n$ with $(n, q)=1$, we have the identity

$$
\sum_{\chi \bmod q}^{*}\left|\sum_{a=1}^{q} \chi\left(a^{2}+n a\right)\right|^{2 k}=J(q) \cdot q^{k} \cdot \prod_{p \| q}\left(1-\frac{1}{p-2}+\frac{1}{p^{k}(p-2)}\right),
$$

where $\sum_{x \bmod q}^{*}$ denotes the summation over all primitive characters $\bmod q, p^{\alpha} \| q$ denotes that $p^{\alpha} \mid q$ and $p^{\alpha+1} \nmid q$, and $J(q)=\sum_{d \mid q} \mu(d) \phi\left(\frac{q}{d}\right)$ denotes the number of all primitive characters $\bmod q$.

Theorem 2 Let $q>1$ be an odd integer. Then, for any real number $k$ and integer $n$ with $(n, q)=1$, we have the identity

$$
\begin{aligned}
\sum_{\chi \bmod q}^{*}\left|\sum_{a=1}^{q} \chi\left(a^{3}+n a^{2}\right)\right|^{2 k}= & J(q) \cdot q^{k} \cdot \prod_{p \| q, 3 \nmid p-1}\left(1-\frac{1}{p-2}+\frac{1}{p^{k}(p-2)}\right) \\
& \times \prod_{p \| q, 3 \mid p-1}\left(1-\frac{3}{p-2}+\frac{3}{p^{k}(p-2)}\right) .
\end{aligned}
$$

From these two theorems we may immediately deduce the following corollaries.

Corollary 1 Let $q$ be an odd square-full number (that is, for any prime $p$, if $p \mid q$, then $\left.p^{2} \mid q\right)$. Then, for any real number $k$ and integer $n$ with $(n, q)=1$, we have the identity

$$
\sum_{\chi \bmod q}^{*}\left|\sum_{a=1}^{q} \chi\left(a^{2}+n a\right)\right|^{2 k}=\sum_{\chi \bmod q}^{*}\left|\sum_{a=1}^{q} \chi\left(a^{3}+n a^{2}\right)\right|^{2 k}=J(q) \cdot q^{k}
$$

Corollary 2 Let $q$ be an odd square-free number (that is, for any prime $p, p^{2} \dagger q$ ). Then, for any real number $k$ and integer $n$ with $(n, q)=1$, we have the identity

$$
\sum_{\chi \bmod q}^{*}\left|\sum_{a=1}^{q} \chi\left(a^{2}+n a\right)\right|^{2 k}=q^{k} \cdot \prod_{p \mid q}\left(p-3+\frac{1}{p^{k}}\right) .
$$

Corollary 3 Let $q$ be an odd square-full number. Then, for any integer $n$ with $(n, q)=1$, we have the identity

$$
\sum_{\chi \bmod q}^{*}\left|\sum_{a=1}^{q} \chi\left(a^{2}+n a\right)\right|^{-2}=\sum_{\chi \bmod q}^{*}\left|\sum_{a=1}^{q} \chi\left(a^{3}+n a^{2}\right)\right|^{-2}=\frac{J(q)}{q} .
$$


For general characters $\chi$ mod $q$, whether there exists an identity for

$$
\sum_{\chi \bmod q}\left|\sum_{a=1}^{q} \chi\left(a^{r}+n a^{s}\right)\right|^{2 k}
$$

is an interesting open problem, where $r>s$ are two positive integers.

\section{Several lemmas}

In this section, we shall give several lemmas, which are necessary in the proof of our theorems. Hereinafter, we shall use many properties of character sums and Gauss sums, all of which can be found in references [5] and [6], so they will not be repeated here. First we have the following lemmas.

Lemma 1 Let $q>1$ be an integer, $J(q)$ denotes the number of all primitive characters mod $q$. Then we have the identity

$$
J(q)=\sum_{d \mid a} \mu(d) \cdot \phi\left(\frac{q}{d}\right)
$$

where $\mu(n)$ is the Möbius function, $\phi(n)$ is the Euler function.

Proof This is a well-known result, here we give a simple proof. It is clear that for any nonprincipal character $\chi \bmod q$, there exists one and only one $d \mid q$ and a primitive character $\chi^{*} \bmod d$ such that $\chi=\chi^{*} \cdot \chi_{0}$, where $\chi_{0}$ denotes the principal character $\bmod q$. So, from these properties, we have

$$
\phi(q)=\sum_{\chi \bmod q} 1=\sum_{d \mid q} J(d) .
$$

From this identity and the Möbius inversion formula, we may immediately deduce

$$
J(q)=\sum_{d \mid a} \mu(d) \cdot \phi\left(\frac{q}{d}\right)
$$

This proves Lemma 1.

Lemma 2 Let $p$ be an odd prime, $i \geq 2$ be an integer and $q=p^{i}$. Then, for any real number $k$ and integer $n$ with $(n, q)=1$, we have the identities

$$
\sum_{\chi \bmod q}^{*}\left|\sum_{a=1}^{q} \chi\left(a^{2}+n a\right)\right|^{2 k}=\sum_{\chi \bmod q}^{*} q^{k}=\phi^{2}(q) \cdot q^{k-1}
$$

and

$$
\sum_{\chi \bmod p}^{*}\left|\sum_{a=1}^{p} \chi\left(a^{2}+n a\right)\right|^{2 k}=1+(p-3) \cdot p^{k} .
$$


Proof For any primitive character $\chi \bmod q$, from the properties of Gauss sums, we know that

$$
\begin{aligned}
\sum_{a=1}^{q} \chi\left(a^{2}+n a\right) & =\frac{1}{\tau(\bar{\chi})} \sum_{a=1}^{q} \sum_{b=1}^{q} \bar{\chi}(b) e\left(\frac{b\left(a^{2}+n a\right)}{q}\right) \\
& =\frac{1}{\tau(\bar{\chi})} \sum_{a=1}^{q} \sum_{b=1}^{q} \bar{\chi}(b \bar{a}) e\left(\frac{b \bar{a}\left(a^{2}+n a\right)}{q}\right) \\
& =\frac{1}{\tau(\bar{\chi})} \sum_{b=1}^{q} \bar{\chi}(b) e\left(\frac{n b}{q}\right) \sum_{a=1}^{q} \chi(a) e\left(\frac{b a}{q}\right) \\
& =\frac{\tau(\chi)}{\tau(\bar{\chi})} \sum_{b=1}^{q} \bar{\chi}^{2}(b) e\left(\frac{n b}{q}\right) \\
& =\chi^{2}(n) \frac{\tau(\chi) \cdot \tau\left(\bar{\chi}^{2}\right)}{\tau(\bar{\chi})} .
\end{aligned}
$$

Note that $q=p^{i}$ and $i \geq 2$, so if $\chi$ is a primitive character $\bmod q$, then $\bar{\chi}^{2}$ is also a primitive character $\bmod q$, so that $|\tau(\chi)|=|\tau(\bar{\chi})|=\left|\tau\left(\bar{\chi}^{2}\right)\right|=\sqrt{q}$. From these identities, Lemma 1 and formula (4), we may immediately deduce that

$$
\sum_{\chi \bmod q}^{*}\left|\sum_{a=1}^{q} \chi\left(a^{2}+n a\right)\right|^{2 k}=\sum_{\chi \bmod q}^{*} q^{k}=\phi^{2}(q) \cdot q^{k-1} .
$$

This proves the first formula of Lemma 2 .

Now we prove the second formula. If $\chi$ is the Legendre symbol mod $p$, then we have $\left|\tau\left(\bar{\chi}^{2}\right)\right|=1$. So, from Lemma 1 and the method of proving (4), we have

$$
\sum_{\chi \bmod p}^{*}\left|\sum_{a=1}^{p} \chi\left(a^{2}+n a\right)\right|^{2 k}=1+(p-3) p^{k}
$$

This completes the proof of Lemma 2.

Lemma 3 Let $p>3$ be an odd prime, $\alpha \geq 2$ be an integer and $q=p^{\alpha}$. Then, for any real number $k$ and $n$ with $(n, q)=1$, we have the identities

$$
\sum_{\chi \bmod q}^{*}\left|\sum_{a=1}^{q} \chi\left(a^{3}+n a^{2}\right)\right|^{2 k}=\phi^{2}(q) \cdot q^{k-1}
$$

and

$$
\sum_{\chi \bmod p}^{*}\left|\sum_{a=1}^{p} \chi\left(a^{3}+n a^{2}\right)\right|^{2 k}= \begin{cases}3+(p-5) \cdot p^{k} & \text { if } p \equiv 1 \bmod 3 \\ 1+(p-3) \cdot p^{k} & \text { if }(3, p-1)=1 .\end{cases}
$$


Proof From the properties of primitive characters $\bmod q$ and the method of proving Lemma 2, we have

$$
\begin{aligned}
\sum_{a=1}^{q} \chi\left(a^{3}+n a^{2}\right) & =\frac{1}{\tau(\bar{\chi})} \sum_{a=1}^{q} \sum_{b=1}^{q} \bar{\chi}(b) e\left(\frac{b\left(a^{3}+n a^{2}\right)}{q}\right) \\
& =\frac{1}{\tau(\bar{\chi})} \sum_{b=1}^{q} \bar{\chi}(b) e\left(\frac{n b}{q}\right) \sum_{a=1}^{q} \chi^{2}(a) e\left(\frac{b a}{q}\right) \\
& =\chi^{3}(n) \frac{\tau\left(\chi^{2}\right) \cdot \tau\left(\bar{\chi}^{3}\right)}{\tau(\bar{\chi})} .
\end{aligned}
$$

Since $\chi$ is a primitive character $\bmod q$ and $(3, q)=1$, so $\chi^{2}$ and $\bar{\chi}^{3}$ are also two primitive characters mod $q$. So, from Lemma 1, (5) and the properties of Gauss sums, we can deduce the first identity of Lemma 3.

Note that if $(3, p-1)=1, \chi_{1}$ is the Legendre symbol $\bmod p$, then for any non-principal character $\chi \bmod p$, we have $\left|\tau\left(\bar{\chi}^{3}\right)\right|=\sqrt{p}$ and $\left|\tau\left(\chi_{1}^{2}\right)\right|=1$. So, from Lemma 1 and the method of proving (5), we have

$$
\sum_{\chi \bmod p}^{*}\left|\sum_{a=1}^{p} \chi\left(a^{3}+n a^{2}\right)\right|^{2 k}=1+(p-3) p^{k}
$$

If $3 \mid p-1$, then there exist two 3 -order characters $\bmod p$, so from the method of proving (6), we have the identity

$$
\sum_{\chi \bmod p}^{*}\left|\sum_{a=1}^{p} \chi\left(a^{3}+n a^{2}\right)\right|^{2 k}=3+(p-5) p^{k} .
$$

Combining (6) and (7), we can deduce the second identity of Lemma 3.

Lemma 4 Let $q_{1}>1$ and $q_{2}>1$ be two integers with $\left(q_{1}, q_{2}\right)=1, \chi_{1} \bmod q_{1}$ and $\chi_{2} \bmod q_{2}$. Then, for any integer $n$ with $\left(n, q_{1} q_{2}\right)=1$, we have

$$
\left|\sum_{a=1}^{q_{1} q_{2}} \chi_{1} \chi_{2}\left(a^{2}+n a\right)\right|=\left|\sum_{a=1}^{q_{1}} \chi_{1}\left(a^{2}+n a\right)\right| \cdot\left|\sum_{b=1}^{q_{2}} \chi_{1}\left(b^{2}+n b\right)\right| .
$$

Proof From the properties of complete residue system $q_{1} q_{2}$, we have

$$
\begin{aligned}
& \left|\sum_{a=1}^{q_{1} q_{2}} \chi_{1} \chi_{2}\left(a^{2}+n a\right)\right| \\
& \quad=\left|\sum_{a=1}^{q_{1}} \sum_{b=1}^{q_{2}} \chi_{1} \chi_{2}\left(\left(a q_{2}+b q_{1}\right)^{2}+n\left(a q_{2}+b q_{1}\right)\right)\right| \\
& \quad=\left|\sum_{a=1}^{q_{1}} \chi_{1}\left(\left(a q_{2}+b q_{1}\right)^{2}+n\left(a q_{2}+b q_{1}\right)\right) \sum_{b=1}^{q_{2}} \chi_{2}\left(\left(a q_{2}+b q_{1}\right)^{2}+n\left(a q_{2}+b q_{1}\right)\right)\right| \\
& \quad=\left|\sum_{a=1}^{q_{1}} \chi_{1}\left(\left(a q_{2}\right)^{2}+n\left(a q_{2}\right)\right) \sum_{b=1}^{q_{2}} \chi_{2}\left(\left(b q_{1}\right)^{2}+n\left(b q_{1}\right)\right)\right|
\end{aligned}
$$




$$
\begin{aligned}
& =\left|\sum_{a=1}^{q_{1}} \chi_{1}\left(a^{2}+n a\right) \sum_{b=1}^{q_{2}} \chi_{2}\left(b^{2}+n b\right)\right| \\
& =\left|\sum_{a=1}^{q_{1}} \chi_{1}\left(a^{2}+n a\right)\right| \cdot\left|\sum_{b=1}^{q_{2}} \chi_{1}\left(b^{2}+n b\right)\right| .
\end{aligned}
$$

This proves Lemma 4.

\section{Proof of the theorems}

In this section, we shall complete the proof of our theorems. First we prove Theorem 1 . For any odd number $q>1$, it is clear that there exist two integers $M$ and $N$ such that $q=M \cdot N$, where $M$ is a square-free number, and $N$ is a square-full number. Now, for any primitive character $\chi \bmod q$, there exist two primitive characters $\chi_{1} \bmod M$ and $\chi_{2} \bmod N$ such that $\chi=\chi_{1} \chi_{2}$. Note that $J(N)=\frac{\phi^{2}(N)}{N}$, so from these properties, Lemma 1, Lemma 2 and Lemma 4 , we have

$$
\begin{aligned}
& \sum_{\chi \bmod q}^{*}\left|\sum_{a=1}^{q} \chi\left(a^{2}+n a\right)\right|^{2 k} \\
& =\left(\sum_{\chi \bmod M}^{*}\left|\sum_{a=1}^{M} \chi\left(a^{2}+n a\right)\right|^{2 k}\right) \cdot\left(\sum_{\chi \bmod N}^{*}\left|\sum_{a=1}^{N} \chi\left(a^{2}+n a\right)\right|^{2 k}\right) \\
& =\prod_{p \mid M}\left(1+(p-3) \cdot p^{k}\right) \prod_{p^{\alpha} \| N}\left(\phi^{2}\left(p^{\alpha}\right) \cdot p^{\alpha(k-1)}\right) \\
& =J(q) \cdot q^{k} \cdot \prod_{p \| q}\left(1-\frac{1}{p-2}+\frac{1}{p^{k}(p-2)}\right),
\end{aligned}
$$

where $p^{\alpha} \| q$ denotes that $p^{\alpha} \mid q$ and $p^{\alpha+1} \nmid q$. This proves Theorem 1 .

Now we prove Theorem 2. From Lemma 3, Lemma 4 and the method of proving Theorem 1 , we have

$$
\begin{aligned}
& \sum_{\chi \bmod q}^{*}\left|\sum_{a=1}^{q} \chi\left(a^{3}+n a^{2}\right)\right|^{2 k} \\
& \left.=\sum_{\chi \bmod M}\left|\sum_{a=1}^{M} \chi\left(a^{3}+n a^{2}\right)\right|^{2 k}\right) \cdot\left(\sum_{\chi \bmod N}^{*}\left|\sum_{a=1}^{N} \chi\left(a^{3}+n a^{2}\right)\right|^{2 k}\right) \\
& =\prod_{p \mid M}\left(1+(p-3) \cdot p^{k}\right) \prod_{p^{\alpha} \| N}\left(\phi^{2}\left(p^{\alpha}\right) \cdot p^{\alpha(k-1)}\right) \\
& =J(q) \cdot q^{k} \cdot \prod_{p \| q, 3 \nmid p-1}\left(1-\frac{1}{p-2}+\frac{1}{p^{k}(p-2)}\right) \\
& \quad \times \prod_{p \| q, 3 \mid p-1}\left(1-\frac{3}{p-2}+\frac{3}{p^{k}(p-2)}\right) .
\end{aligned}
$$

This completes the proof of our theorems. 
Competing interests

The authors did not provide this information.

\section{Authors' contributions}

The authors did not provide this information.

\section{Acknowledgements}

The authors would like to thank the referee for their very helpful and detailed comments, which have significantly improved the presentation of this paper. This work is supported by the National Natural Science Foundation of P.R. China (No. 11071194)

Received: 6 July 2013 Accepted: 17 October 2013 Published: 12 Nov 2013

\section{References}

1. Burgess, DA: On Dirichlet characters of polynomials. Proc. Lond. Math. Soc. 13, 537-548 (1963)

2. Granville, A, Soundararajan, K: Large character sums: pretentious characters and the Pólya-Vinogradov theorem. J. Am. Math. Soc. 20, 357-384 (2007)

3. Wenpeng, Z, Yuan, Y: On Dirichlet characters of polynomials. Bull. Lond. Math. Soc. 34, 469-473 (2002)

4. Wenpeng, Z, Weili, Y: A note on the Dirichlet characters of polynomials. Acta Arith. 115, 225-229 (2004)

5. Apostol, TM: Introduction to Analytic Number Theory. Springer, New York (1976)

6. Chengdong, P, Chengbiao, P: Goldbach Conjecture. Science Press, Beijing (1992)

10.1186/1029-242X-2013-533

Cite this article as: Guo and Wang: An identity involving the mean value of two-term character sums. Journal of Inequalities and Applications 2013, 2013:533

\section{Submit your manuscript to a SpringerOpen ${ }^{\circ}$ journal and benefit from:}

- Convenient online submission

- Rigorous peer review

- Immediate publication on acceptance

Open access: articles freely available online

- High visibility within the field

- Retaining the copyright to your article 